\title{
Efeito da ingestão de fumonisina $B$ no peso corporal e na histopatologia de codornas japonesas (Coturnix coturnix japonica)
}

\author{
Effect of fumonisin $B_{1}$ on body weight and histopathology of Japanese quail \\ (Coturnix coturnix japonica)
}

\author{
Carlos Augusto Fernandes Oliveira ${ }^{\mathrm{I}}$ Paula Butkeraitis ${ }^{\mathrm{I}}$ \\ David Randolph Ledoux ${ }^{\text {II }}$ George Rottinghaus ${ }^{\text {II }}$
}

\begin{abstract}
- NOTA -
RESUMO

O objetivo do presente trabalho foi estudar o efeito da fumonisina $B_{1}\left(F B_{1}\right)$ sobre o peso corpóreo e as vísceras (fígado, rim e coração) de codorna poedeira japonesa (Coturnix coturnix japonica), devido ao escasso dado toxicológico nesta espécie. Quatro grupos, sendo cada um constituído de 32 codornas de linhagem comercial, receberam

$(P<0.05)$. Relative weights of kidney and heart were similar $(P>0.05)$ among treatments. However, the relative weight of liver increased $(P<0.05)$ in quail of groups received 50 and $250 \mathrm{mg} \mathrm{FB} / \mathrm{kg}$. Histological changes were observed only in the liver of groups receiving 50 and $250 \mathrm{mgFB}_{1} \mathrm{~kg}^{-1}$, which caused moderate biliary duct hyperplasia and multiple foci of hepatic necrosis. The data indicated that AFB at levels above $50 \mathrm{mg}$ $\mathrm{kg}^{-1}$ in laying Japanese quail.
\end{abstract} ração contendo $F B$ nas concentrações de 0 (controle), 10, 50 e $250 \mathrm{mg} \mathrm{kg}^{-1}$, durante 28 dias. Observou-se uma redução $(P<0,05)$ no peso corpóreo das aves submetidas ao tratamento $250 \mathrm{mg} \mathrm{kg}^{-1}$ de $F B_{1}$. Os pesos relativos do rim e do coração mantiveram-se semelhantes em todos os tratamentos $(P>0,05)$, porém as aves do grupo 50 e $250 \mathrm{mg} \mathrm{kg}^{-1}$ apresentaram aumento no peso relativo de fígado $(P<0,05)$. Alterações histológicas foram constatadas apenas no fígado das aves alimentadas com ração contendo 50 e $250 \mathrm{mg} \mathrm{kg}^{-1}$ de $F B_{1}$, caracterizadas por hiperplasia moderada de ductos biliares $e$ múltiplas áreas de necrose focal, indicando que a $F B_{1}$ seja tóxica a partir de $50 \mathrm{mg} \mathrm{kg}^{-1}$ para codornas de postura.

Palavras-chave: $F B_{1}$, toxicidade, alterações histopatológicas, codorna.

\section{ABSTRACT}

This research was aimed at evaluating the effect of fumonisin $B_{1}\left(F B_{1}\right)$ on body weight and viscera (liver, kidney and heart) of laying Japanese quail (Coturnix coturnix japonica), due to the little toxicological data on this species. Four experimental groups of 32 commercial quails were designed and exposed to ingestion test with feed containing 0 (controls), 10, 50 or $250 \mathrm{mg} \mathrm{FB}_{1} \mathrm{~kg}^{-1}$ feed, during 28 days. Birds of group 250mg $\mathrm{FB}_{1} \mathrm{~kg}^{-1}$ showed lower body weight
Key words: $F B_{1}$, toxicity, histopathology, quail.

As fumonisinas são produzidas por fungos do gênero Fusarium, principalmente da espécie $\boldsymbol{F}$. verticillioides (Sacc.) Nirenberg (=F. moniliforme Sheldon, teleomorfo Giberella fujikuroi Sawada) (RHEEDER et al., 2002). Mais de 28 diferentes tipos de fumonisinas já foram isolados, sendo a toxina predominante a fumonisina $\mathrm{B}_{1}\left(\mathrm{FB}_{1}\right)$, que também apresenta maior toxicidade. $\mathrm{AFB}_{1}$ tem sido associada com baixo desempenho, aumento do peso relativo de vísceras e hepatite tóxica em aves domésticas (KUBENA et al., 1995). LEDOUX et al. (1992) observaram diarréia, diminuição do consumo de alimentos e do ganho de peso, aumento do peso do fígado, necrose hepática e hiperplasia hepatocelular em frango de corte alimentado com ração contendo 100-400mg kg-1 de FB $\mathrm{FB}_{1}$ Alterações de miocárdio,

IFaculdade de Zootecnia e Engenharia de Alimentos, Universidade de São Paulo (USP), Av. Duque de Caxias Norte 225, 13635-900, Pirassununga, SP, Brasil. E-mail: carlosaf@usp.br. Autor para correspondência.

${ }^{\text {II } C o l l e g e ~ o f ~ A g r i c u l t u r e, ~ F o o d ~ a n d ~ N a t u r a l ~ R e s o u r c e s, ~ U n i v e r s i t y ~ o f ~ M i s s o u r i, ~ C o l u m b i a, ~ M O, ~ U n i t e d ~ S t a t e s . ~}$

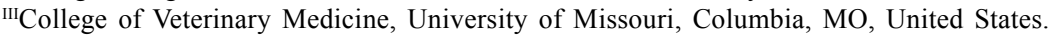


caracterizadas por perda de estriações transversais e adelgaçamento dos cardiomiócitos foram relatados em perus alimentados com níveis de $\mathrm{FB}_{1}$ acima de $325 \mathrm{mg}$ $\mathrm{kg}^{-1}$ (WEIBKING et al., 1994).

Com relação às codornas, níveis de $\mathrm{FB}_{1}$ de 50 e $100 \mathrm{mg} \mathrm{kg}^{-1}$ causaram redução no consumo de ração, diminuição do peso corpóreo, redução do peso e despigmentação dos ovos e redução do peso da casca (BUTKERAITIS et al., 2004). O objetivo do presente trabalho foi verificar os efeitos da $\mathrm{FB}_{1}$ sobre o peso corpóreo, fígado, rins e coração de codornas poedeiras alimentadas com rações contendo diferentes níveis da toxina.

Foram utilizadas 128 codornas de linhagem comercial Japonesa (Coturnix coturnix japonica), adquiridas com cinco semanas de idade e vacinadas, subdivididas em quatro grupos de 32 aves. As aves foram mantidas em adaptação por duas semanas, sendo alimentadas com ração convencional à base de milho $\mathrm{e}$ farelo de soja, contendo $\mathrm{FB}_{1}$ abaixo de $1,0 \mathrm{mg} \mathrm{kg}^{-1}$, conforme as recomendações do NATIONAL RESEARCH COUNCIL (1994). As aves foram alojadas em bateria contendo quatro gaiolas de arame providas de comedouros lineares e bebedouros tipo calha em "V" para água corrente.

A FB ${ }_{1}$ utilizada no experimento foi produzida a partir do cultivo de cepa toxigênica de $\boldsymbol{F}$. verticillioides (cepa $\mathrm{M}-1325^{\mathrm{a}}$ ) e mantida em material de cultivo à base de milho, homogeneizado e esterilizado, conforme os procedimentos descritos por WEIBKING et al. (1994). O material de cultivo foi preparado em jarras de $1 \mathrm{~L}$, onde $100 \mathrm{~g}$ de grãos de milho e $100 \mathrm{~mL}$ de água destilada foram autoclavados $\left(120^{\circ} \mathrm{C} /\right.$ $30 \mathrm{~min}$ ) e, em seguida, semeados com $2 \mathrm{~mL}$ de água destilada contendo a cepa de $\boldsymbol{F}$. verticillioides. As jarras foram agitadas manualmente e incubadas a $27^{\circ} \mathrm{C}$ por cinco semanas. Após a incubação, adicionou-se $400 \mathrm{~mL}$ de acetona:clorofórmio (75:25) ao meio de cultura, seguido de agitação em homogeneizador de alta velocidade por $5 \mathrm{~min}$. Após a filtração, o resíduo sólido foi submetido a secagem em estufa a $40^{\circ} \mathrm{C}$ e triturado em um moinho de disco até a obtenção de um pó fino.

A concentração de fumonisinas no material de cultivo em pó foi determinada através de cromatografia líquida de alta eficiência (CLAE) $\left(\right.$ Shimadzu $\left.{ }^{\circledR}\right)$ com detecção por fluorescência (comprimentos de onda de 335 e 440nm para excitação e emissão, respectivamente), utilizando-se a técnica descrita por SHEPHARD et al. (1990). A extração foi realizada tomando-se $50 \mathrm{~g}$ da amostra e adicionando-se
$50 \mathrm{~mL}$ de acetonitrila:água (1:1). Após agitação por 30min, a mistura foi filtrada, transferindo-se $2 \mathrm{~mL}$ do filtrado para coluna de extração em fase sólida (SPE), previamente condicionada com $2 \mathrm{~mL}$ de metanol e $2 \mathrm{~mL}$ de água destilada. Após a lavagem inicial com $2 \mathrm{~mL}$ de acetonitrila:água (20:80), o extrato foi eluído com $2 \mathrm{~mL}$ de acetonitrila:água (70:30). Em seguida, uma alíquota de $200 \mu \mathrm{L}$ do extrato final foi misturada com $200 \mu \mathrm{L}$ de reativo OPA, previamente preparado com $40 \mu \mathrm{g}$ de ophthaldialdehydo em $5 \mathrm{~mL}$ de solução de tetraborato de sódio a $0,1 \mathrm{M}$ e $50 \mu \mathrm{L}$ de 2-mercaptohetanol. Após dois minutos, foram aplicados $20 \mu \mathrm{L}$ no sistema CLAE, utilizando-se coluna de fase reversa $\mathrm{C} 18$, com dimensões de $150 \mathrm{X} 4,6 \mathrm{~mm}$ e tamanho de partícula $5 \mu \mathrm{m}$ $\left(\right.$ Phenomenex $\left.{ }^{\circledR}\right)$. A fase móvel foi composta por acetonitrila:água:ácido acético (50:50:1), empregada com fluxo constante de $1 \mathrm{~mL} \mathrm{~min}^{-1}$. Os limites de quantificação do método para FB1 e FB2 foram de 20 e $30 \mu \mathrm{g} \mathrm{kg}^{-1}$, respectivamente. As concentrações de FB1 e FB2 no material de cultivo foram de $6.500 \mathrm{mg} \mathrm{kg}^{-1} \mathrm{e}$ $2.100 \mathrm{mg} \mathrm{kg}^{-1}$, respectivamente.

A adição de material de cultivo contendo FB1 à ração $\left(0,2-3,0 \mathrm{~kg} 100 \mathrm{~kg}^{-1}\right.$ de ração) foi realizada em um misturador horizontal/helicoidal (Marconi ${ }^{\circledR}$ ), em uma única batida, um dia antes do início do período experimental, totalizando cerca de $90 \mathrm{~kg}$ de ração. Utilizou-se a mesma ração empregada no período de adaptação das aves, constituída à base de milho e farelo de soja, balanceada conforme o NATIONAL RESEARCH COUNCIL (1994). A confirmação dos níveis de $\mathrm{FB}_{1}$ nas rações experimentais foi realizada de acordo com a mesma técnica descrita acima, com quantificação através de CLAE (SHEPHARD et al., 1990). Após a análise das rações preparadas, os níveis de $\mathrm{FB}_{1}$ nos tratamentos que continham 10,50 e $250 \mathrm{mg} / \mathrm{kg}$ foram de $8,6 \mathrm{mg} \mathrm{kg}^{-1}, 53,5 \mathrm{mg} \mathrm{kg}^{-1}$ e $254,2 \mathrm{mg} \mathrm{kg}^{-1}$, respectivamente. As rações de cada batida também foram analisadas quanto à presença de aflatoxinas, ocratoxina A e zearalenona, através de cromatografia de camada delgada (SOARES \& RODRIGUEZ-AMAYA, 1989), sendo que nenhuma delas foi detectada nas amostras. Os limites de quantificação do método de análise para aflatoxinas, ocratoxina A e zearalenona foram de 4,0 $\mu \mathrm{g}$ $\mathrm{kg}^{-1}, 5,0 \mu \mathrm{g} \mathrm{kg}^{-1}$ e $55,0 \mu \mathrm{g} \mathrm{kg}^{-1}$, respectivamente.

$\mathrm{O}$ experimento com intoxicação iniciou-se com aves com sete semanas de idade, no qual as aves receberam diariamente cerca de $30 \mathrm{~g}$ ração ave ${ }^{-1}$ e água ad libitum durante 28 dias. Após o período, as aves foram pesadas, anestesiadas com éter etílico e sacrificadas por deslocamento cervical e imediatamente 
necropsiadas, para análise de vísceras (pesagem e exame macroscópico de possíveis lesões). Tecidos do fígado, rim e coração foram recolhidos em frascos contendo formalina a $10 \%$ tamponada e submetidos ao exame histopatológico (VASCONCELOS, 1988). Secções representativas de tecidos (espessura de $5 \mu \mathrm{m}$ ) foram coradas com hematoxilina-eosina e examinadas ao microscópio de luz. O peso absoluto de vísceras foi convertido para peso relativo $\left(\mathrm{g} 100 \mathrm{~g}^{-1}\right.$ de peso corpóreo - pc) e submetido à análise de variância (SAS, 1992), comparando-se as médias através do teste de Tukey $(\mathrm{P}<0,05)$.

O peso corporal das aves no dia 1 de intoxicação e imediatamente após o sacrifício (dia 28), bem como os pesos relativos do fígado, rim e coração, encontram-se na tabela 1. Aos 28 dias, observou-se uma redução $(\mathrm{P}<0,05)$ no peso corpóreo das aves submetidas ao tratamento com $200 \mathrm{mg} \mathrm{kg}^{-1}$ de $\mathrm{FB}_{1}$, em relação aos demais tratamentos. Resultados semelhantes foram observados em patos Pekin intoxicados com 100-400mg $\mathrm{kg}^{-1}$ de $\mathrm{FB}_{1}$ (BERMUDEZ et al., 1995) e em perus alimentados com ração contendo $75-425 \mathrm{mg} \mathrm{kg}^{-1}$ (KUBENA et al., 1995). Embora o mecanismo de ação de fumonisinas ainda não seja totalmente elucidado, a hipótese mais aceita consiste na interferência no metabolismo dos esfingolipídeos, devido à inibição da enzima N-aciltransferase (WANG, 1991). STEVENS \& TANG (1997) demonstraram que a concentração reduzida de esfingolipídeos, ocasionada pela exposição à $\mathrm{FB}_{1}$, promoveu o bloqueio quase completo do transporte nos receptores folato, o que pode levar ao comprometimento de processos celulares dependentes dessa vitamina, com conseqüente redução do ganho de peso corpóreo.

Os pesos de rim e coração mantiveram-se semelhantes $(\mathrm{P}>0,05)$ em todos os tratamentos, em relação ao controle. No entanto, o peso de fígado de aves intoxicadas com $\mathrm{FB}_{1}$ apresentou-se maior em relação ao controle, sendo a diferença significativa
$(\mathrm{P}<0,05)$ somente para os grupos 50 e $250 \mu \mathrm{g} \mathrm{kg}^{-1}$. Estes resultados concordam com os dados reportados sobre os efeitos da $\mathrm{FB}_{1}$ sobre o fígado de outras espécies de aves domésticas, a exemplo de frango de corte (LEDOUX et al., 1992) e peru (WEIBKING et al., 1994). $\mathrm{O}$ aumento no peso hepático, observado em todas as espécies de aves estudadas, parece estar associado com as alterações no metabolismo lipídico promovidas pela fumonisina (KUBENA et al., 1995).

As amostras de rim e coração de ave controle, assim como das intoxicadas com 10, $50 \mathrm{e}$ $250 \mathrm{mg} \mathrm{kg}^{-1}$ de $\mathrm{FB}_{1}$, não apresentaram alterações significativas $(\mathrm{P}>0,05)$. Com relação ao fígado, as amostras provenientes dos grupos controle e $10 \mathrm{mg}$ $\mathrm{kg}^{-1}$ de $\mathrm{FB}_{1}$ também não apresentaram lesões aparentes; porém, algumas áreas isoladas apresentaram-se com discreta degeneração vacuolar do tecido hepático. RANDALL \& REECE (1996) também descreveram relato semelhante, relacionando o fato acima, provavelmente, à esteatose fisiológica observada em praticamente todas as aves em postura.

Os fígados de aves alimentadas com 50 e $250 \mathrm{mg} \mathrm{kg}^{-1}$ de $\mathrm{FB}_{1}$ apresentaram hiperplasia moderada de ductos biliares e múltiplas áreas de necrose focal. A severidade das lesões hepáticas foi maior nas aves alimentadas com $250 \mathrm{mg} \mathrm{kg}^{-1}$, em relação ao grupo que recebeu $50 \mathrm{mg} \mathrm{kg}^{-1}$, indicando um efeito proporcional à dose de $\mathrm{FB}_{1}$. Os dados apresentados estão de acordo com os resultados obtidos em frango de corte (LEDOUX et al., 1992) e peru (WEIBKING et al., 1994). Este efeito hepatotóxico pode ser atribuído primariamente, também, à inibição da enzima $\mathrm{N}$-aciltransferase, uma vez que isso determina um desarranjo no metabolismo de esfingolipídeos, incluindo a regulação celular (WANG, 1991).

Os resultados obtidos indicaram que a $\mathrm{FB}_{1}$ adicionada na ração de codornas em concentrações acima $50 \mathrm{mg} \mathrm{kg}^{-1}$ produziu um quadro de hepatite tóxica semelhante ao observado em peru e frango de corte.

Tabela 1 - Efeito da ingestão de $\mathrm{FB}_{1}$ no peso corpóreo e no peso visceral de codornas japonesas ${ }^{1}$.

\begin{tabular}{|c|c|c|c|c|c|}
\hline \multirow{2}{*}{$\begin{array}{c}\mathrm{FB}_{1} \text { na ração } \\
(\mathrm{mg} / \mathrm{kg})\end{array}$} & \multicolumn{2}{|c|}{ Peso corpóreo (g) } & \multicolumn{3}{|c|}{ Pesos relativos (g/100 g de peso corpóreo) } \\
\hline & Dia 1 & Dia 28 & Fígado & $\operatorname{Rim}$ & Coração \\
\hline 0 (controle) & $143,6 \pm 9,8^{\mathrm{a}}$ & $160,0 \pm 1,0^{\mathrm{a}}$ & $2,68 \pm 0,35^{\mathrm{a}}$ & $0,66 \pm 0,14^{\mathrm{a}}$ & $1,00 \pm 0,16^{\mathrm{a}}$ \\
\hline 10 & $145,5 \pm 6,6^{\mathrm{a}}$ & $157,3 \pm 5,7^{\mathrm{a}}$ & $2,98 \pm 0,46^{\mathrm{ab}}$ & $0,71 \pm 0,17^{\mathrm{a}}$ & $1,00 \pm 0,16^{\mathrm{a}}$ \\
\hline 50 & $147,0 \pm 4,8^{\mathrm{a}}$ & $153,7 \pm 4,3^{\mathrm{a}}$ & $3,34 \pm 0,39^{b c}$ & $0,81 \pm 0,19^{\mathrm{a}}$ & $1,04 \pm 0,38^{\mathrm{a}}$ \\
\hline 250 & $145,9+4,3^{\mathrm{a}}$ & $143,3+4,0^{b}$ & $3,55 \pm 0,52^{\mathrm{c}}$ & $0,74 \pm 0,19^{\mathrm{a}}$ & $1,02 \pm 0,16^{\mathrm{a}}$ \\
\hline
\end{tabular}

${ }^{\mathrm{a}, \mathrm{b}} \mathrm{Em}$ uma coluna, médias seguidas de letras iguais não diferem estatisticamente $(\mathrm{P}>0,05)$.

${ }^{1}$ Valores se referem à média \pm desvio padrão para 12 aves em cada tratamento. 


\section{FONTES DE AQUISIÇÃO}

${ }^{a}$ Fusarium Research Center, Pennsylvania State University. University Park, PA, 16802, USA.

\section{REFERÊNCIAS}

BERMUDEZ, A.J. et al. Effects of Fusarium moniliforme culture material containing known levels of fumonisin $\mathrm{B}_{1}$ in ducklings. Avian Diseases, v.39, p.879-886, 1995.

BUTKERAITIS, P. et al. Effect of dietary fumonisin $\mathrm{B}_{1}$ on laying Japanese quail. British Poultry Science, v.45, p.798$801,2004$.

KUBENA, L.F. et al. Effects of feeding Fusarium moniliforme culture material and aflatoxin singly and in combination to turkey polts. Poultry Science, v.74, p.1295-1303, 1995.

LEDOUX, D.R. et al. Fumonisin toxicity in broiler chicks. Journal of Veterinary Diagnostic Investigation, v.4, p.330$333,1992$.

NATIONAL RESEARCH COUNCIL. Nutrient requirements of poultry. 9.ed. Washington : National Academy of Sciences, 1994. 155p.

RANDALL, C.J.; REECE, R.L. Colour atlas of avian histopathology. London : Mosby-Wolfe, 1996. 232p.

RHEEDER, J.P. et al. Production of fumonisin analogs by Fusarium species. Applied Environmental Microbiology, v.68. p.2101-2105, 2002.
SAS Institute. SAS ${ }^{\circledR}$ User's guide: statistics. Cary, NC, 1992. 1686p.

SHEPHARD, G.S. et al. Quantitative determination of fumonisins $B_{1}$ and $B_{2}$ by high-performance liquid chromatography with fluorescence detection. Journal of Liquid Chromatography, v.13, p.2077-2087, 1990

SOARES, L.M.V.; RODRIGUEZ-AMAYA, D.B. Survey of aflatoxins, ochratoxins, zearalenone and sterigmatocystin in some Brazilian foods by using multi-toxin thinlayer chromatographic method. Journal of the Association of Official Analytical Chemists, v.72, p.22-26, 1989.

STEVENS, V.L.; TANG, J. Fumonisin $\mathrm{B}_{1}$ - induced sphingolipid depletion inhibits vitamin uptake via glycosylphosphatidylinositol-anchored folate receptor. Journal of Biological Chemistry, v.272, p.18020-18025, 1997

VASCONCELOS, A.C. Necropsia e remessa de material para laboratório em medicina veterinária. Brasília: ABEAS, 1988. 73p.

WANG, E. et al. Inhibition of sphingolipid biosynthesis by fumonisins. Implications for diseases associated with Fusarium moniliforme. Journal of Biological Chemistry, v.266, p.14486-14490, 1991.

WEIBKING, T.S. et al. Individual and combined effects of feeding Fusarium moniliforme culture material, containing known levels of fumonisin $\mathrm{B}_{1}$, and aflatoxin $\mathrm{B}_{1}$ in the young turkey poult. Poultry Science, v.73, p.1517-1525, 1994. 